\title{
ChemComm
}

Check for updates

Cite this: Chem. Commun., 2017 53, 10188

Received 7th June 2017,

Accepted 29th June 2017

DOI: $10.1039 / c 7 c c 04423 b$

rsc.li/chemcomm

\section{Ultraclean pure shift NMR $\dagger$}

\author{
Pinelopi Moutzouri, (D) ${ }^{a}$ Yingxian Chen, ${ }^{a}$ Mohammadali Foroozandeh, (D) a \\ Peter Kiraly, (D) ${ }^{a}$ Andrew R. Phillips, (D) ${ }^{b}$ Steven R. Coombes, $(\mathbb{D})^{c}$ Mathias Nilsson (D) ${ }^{a}$ \\ and Gareth A. Morris (D) *a
}

\begin{abstract}
"Pure shift" methods can greatly improve the resolution of proton NMR spectra. However, current pure shift spectra show small periodic artefacts that prevent their use for studying dilute mixture components. A new technique, compatible with all current pure shift methods, is presented that suppresses such sidebands to arbitrary order, allowing ultraclean spectra to be obtained.
\end{abstract}

The proton is the most frequently studied nucleus in NMR because of its chemical ubiquity and high sensitivity. ${ }^{1} \mathrm{H}$ spectra are rich in chemical information, but their narrow chemical shift range and extensive overlap of multiplet structure complicate spectral analysis and interpretation. Pure shift $\mathrm{NMR}^{1-3}$ has proven to be a valuable tool for the analysis of crowded spectra, ${ }^{4-6}$ improving resolution by suppressing the effects of homonuclear couplings $J$ while retaining the chemical shift. However, all current direct domain pure shift methods (as opposed to experiments suppressing couplings in an indirect domain of a multidimensional spectrum) suffer from weak periodic artefacts. Such experiments are based on the periodic refocusing of the scalar coupling evolution, using either $1 \mathrm{D}^{7}$ (real-time) or pseudo-2D (interferogram) data acquisition. ${ }^{8}$ The artefacts arise because for sensitivity reasons the pure shift FID is respectively acquired or constructed in chunks of a few tens of ms duration $\left(1 / \mathrm{sw}_{1}\right)$ each, while the scalar couplings are only perfectly refocused at the midpoint of each chunk. ${ }^{8-12}$ The small amount of $J$ evolution during the rest of the chunk causes a weak modulation of the signal envelope with period $1 / \mathrm{sw}_{1}$ (as do a variety of other perturbations, including instrumental imperfections). On Fourier transformation this modulation leads to each peak acquiring small periodic sidebands at intervals $\mathrm{sw}_{1}$. These "chunking

\footnotetext{
${ }^{a}$ School of Chemistry, University of Manchester, Oxford Road, Manchester, M13 9PL, UK. E-mail: g.a.morris@manchester.ac.uk

${ }^{b}$ Pharmaceutical Sciences, AstraZeneca, Silk Road Business Park, Macclesfield, SK10 2NA, UK

${ }^{c}$ Pharmaceutical Technology and Development, AstraZeneca, Silk Road Business Park, Macclesfield, SK10 2NA, UK

$\dagger$ Electronic supplementary information (ESI) available. See DOI: 10.1039/ c7cc04423b
}

sidebands" are typically at a level less than $2-4 \%$ of the parent pure shift signal, and in most cases can be ignored. However, in mixture analysis these artefacts can cause serious problems, obscuring the real signals of minor mixture components. Here, we show that by systematically manipulating the timing of pure shift experiments during normal time averaging it is possible to suppress chunking sidebands to arbitrary order, at negligible cost in sensitivity. This new method (SAPPHIRE, Sideband Averaging by Periodic PHase Incrementation of Residual $J$ Evolution) will allow the measurement of ultraclean pure shift spectra and facilitate the analysis of low-level mixture components.

The most challenging NMR samples are mixtures; those with a wide range of signal intensities are particularly challenging. Fig. 1 shows ${ }^{1} \mathrm{H}$ 1D and pure shift spectra of the anti-cholesterol active pharmaceutical ingredient rosuvastatin (1, Scheme 1), spiked with $2.8 \%$ of its precursor BEM (2, Scheme 1) (see assignment of the pure compounds and their individual ${ }^{1} \mathrm{H}$ 1D spectra in Fig. S3 and S4 of the ESI $\dagger$ ). The conventional ${ }^{1} \mathrm{H}$ pure shift spectrum of Fig. 1b/c, acquired using the interferogrambased Zangger-Sterk method ${ }^{8,13}$ (Fig. S1, ESI $\dagger$ ), is complicated by the presence of chunking sidebands. At the level of $2-4 \%$ of the parent signals, these obscure the BEM signals and severely complicate their analysis. In contrast, the spectrum of Fig. 1d, which was acquired with the new technique described here, almost completely suppresses the sidebands, making the BEM signals clearly visible. The fact that the low-level signals and chunking sidebands are similar in intensity and that the sidebands appear here mostly as negative signals means that overlap between a resonance of interest and a sideband can lead to cancellation. For example, in Fig. 1c the BEM signal resonating at $6.58 \mathrm{ppm}$ is completely suppressed because of its accidental overlap with a negative sideband. In general, the presence of chunking sidebands causes problems with both identification and quantification of low-level signals in the presence of large signals.

There already exists one method for reducing the impact of chunking sidebands, which is simply to average the results of multiple experiments using different values of $\mathrm{sw}_{1}$, spreading 

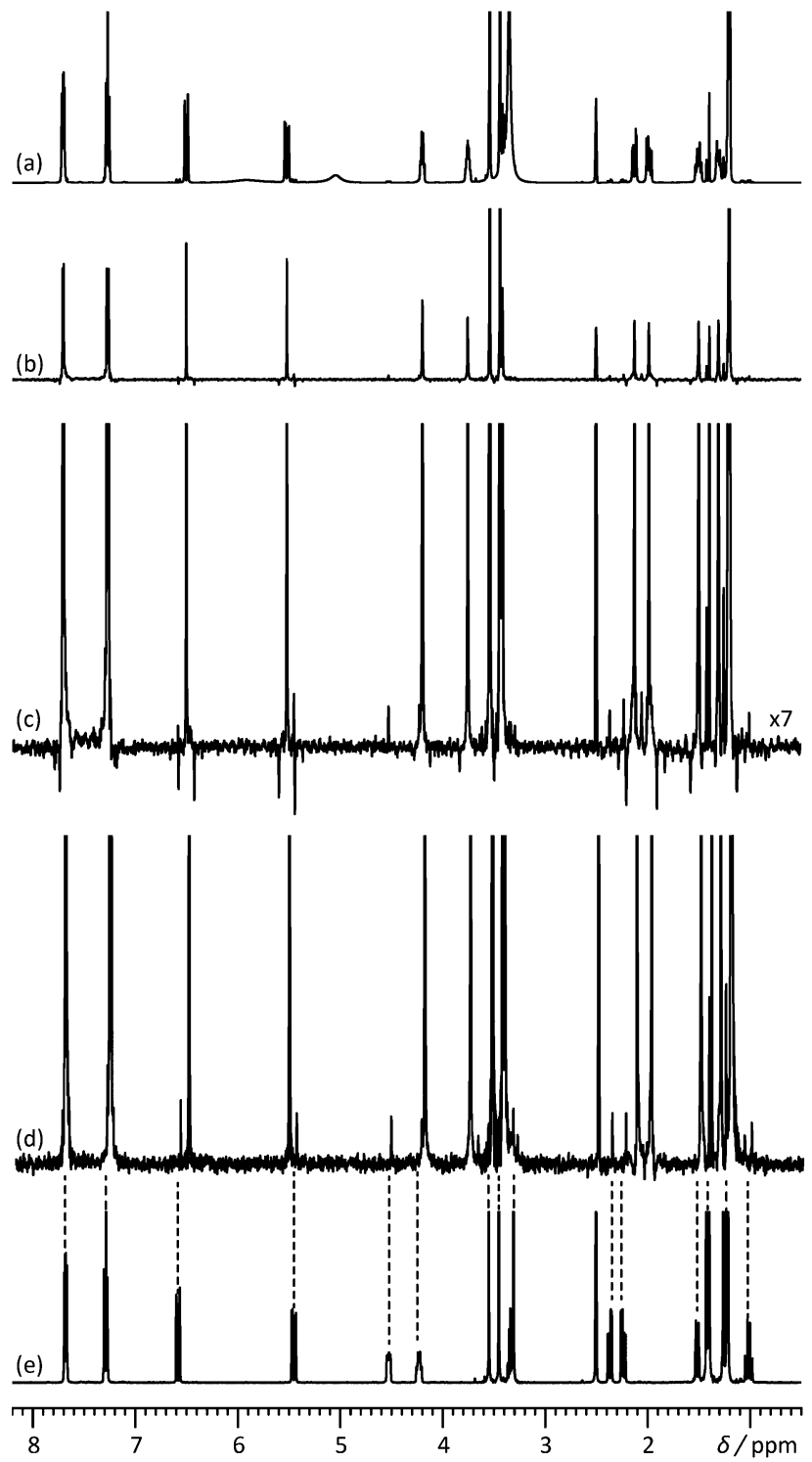

Fig. $1500 \mathrm{MHz}$ (a) ${ }^{1} \mathrm{H} 1 \mathrm{D}$ spectrum, (b) ${ }^{1} \mathrm{H}$ pure shift spectrum recorded using the conventional pulse sequence of Fig. S1 (ESI $\dagger$ ), (c) vertical expansion of the spectrum (b), and (d) ${ }^{1} \mathrm{H}$ pure shift spectrum acquired with the pulse sequence of Fig. 2, for a mixture containing rosuvastatin (1) and 2.8\% of its precursor BEM (2). (e) ${ }^{1} \mathrm{H} 1 \mathrm{D}$ spectrum of BEM. Spectra $\mathrm{c}$ and d were acquired in approximately $9 \mathrm{~h}$ with an sw $\mathrm{sw}_{1}$ of $39.0625 \mathrm{~Hz}$ and 16 chunks using an RSNOB spatially selective spin inversion element with a peak RF amplitude of $47 \mathrm{~Hz}$.
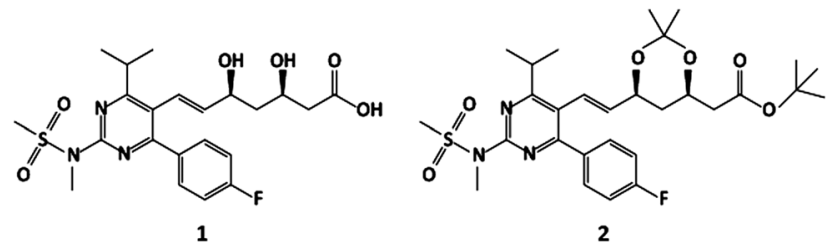

Scheme 1 Rosuvastatin (1) and its precursor BEM (2).

the sidebands out over a range of frequencies, as shown in Fig. S5 of the ESI. $\dagger^{14}$ This method has not yet found significant application, perhaps because it only reduces the peak amplitude of sidebands, but can in favourable cases give sufficient improvement to allow low-level signals to be identified, if not quantified. The new SAPPHIRE method, in contrast, gives essentially complete suppression of sidebands.

Here, we propose a different method that can completely suppress sidebands, by manipulating the phase of the residual modulation through small (ms) timing changes. The classic Zangger-Sterk sequence $^{8}$ in the commonly used form ${ }^{13}$ of Fig. S1 in the ESI $\dagger$ refocuses $J$ modulation at a time $1 /\left(2 \mathrm{sw}_{1}\right)$ after the onset of acquisition, at the midpoint of the chunk of data acquired. Suppose that two experiments are carried out, one with the conventional timing and one in which $J$ is refocused immediately before acquisition. If the results are averaged, the sum of the two modulation patterns will give a pattern in which the peak-to-peak modulation has been reduced and in which the periodicity has halved, from $1 / \mathrm{sw}_{1}$ to $1 /\left(2 \mathrm{sw}_{1}\right)$. Fourier transformation of the averaged signal will then yield a spectrum in which the first sidebands appear at $\pm 2 \mathrm{sw}_{1}$, rather than $\pm \mathrm{sw}_{1}$. As shown in Fig. S8 (ESI $\dagger$ ), extending this to four steps will cancel sidebands up to third order, and so on.

Fig. 2 shows a slightly modified sequence that incorporates a brief extra echo to allow the point of $J$ refocusing to be shifted forwards or backwards in time, so that the $J$ modulation phase can be varied over a complete cycle. The net $J$ evolution time $\tau_{J}$ is $2\left(\tau_{1}-\tau_{3}\right)$, so in the conventional sequence $\tau_{J}=-1 /\left(2 \mathrm{sw}_{1}\right)$. (There is an analogy here with the suppression of sidebands in bilevel heteronuclear decoupling ${ }^{15}$ ). To suppress sidebands to order $N-1$, a series of pure shift interferograms is constructed with $N$ different modulation time shifts $\Delta \tau_{J}$, and subsequently averaged. In acquiring the first chunk of each interferogram, the delays $\tau_{1}$ are of duration $\operatorname{Min}\left(\tau_{\min }+1 /\left(4 \mathrm{sw}_{1}\right)-\Delta \tau_{J} / 2, \tau_{\min }\right)$, the delays $\tau_{3}$ are of duration $\operatorname{Max}\left(\tau_{\min }-1 /\left(4 \mathrm{sw}_{1}\right)+\Delta \tau_{J} / 2, \tau_{\min }\right)$, $\tau_{2}=\tau_{\min }+1 /\left(4 \mathrm{sw}_{1}\right)-\left|\tau_{1}-\tau_{3}\right|$, and the duration of data acquisition (i.e. the size of the first chunk) is $1 / \mathrm{sw}_{1}-\Delta \tau_{J}$, where $\tau_{\min }$ is the minimum time needed for a gradient pulse and subsequent recovery, and $\Delta \tau_{J}$ is incremented from 0 to $(N-1) /\left(N \mathrm{sw}_{1}\right)$ in steps of $1 /\left(N \mathrm{Nw}_{1}\right)$. Subsequent chunks of data in each interferogram are acquired with $\tau_{1}=\tau_{\min }+1 /\left(4 \mathrm{sw}_{1}\right)$ and $\tau_{3}=\tau_{\min }$, but with the evolution time $t_{1}$ reduced by $\Delta \tau_{J}$ to allow for the duration of the first chunk acquired, and $\tau_{2}=\tau_{\min }+\Delta \tau_{J} / 2$.

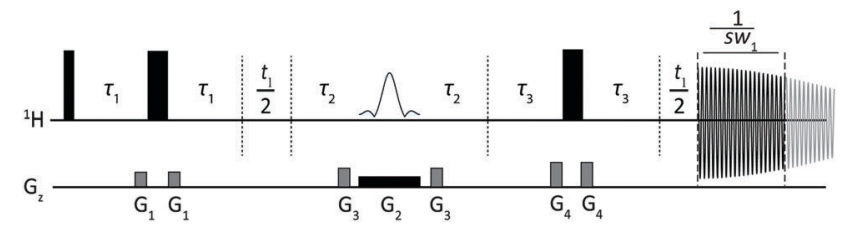

Fig. 2 Modified Zangger-Sterk pulse sequence used here for SAPPHIRE acquisition of clean, sideband-free ${ }^{1} \mathrm{H}$ pure shift spectra. Closed narrow and wide rectangles in the ${ }^{1} \mathrm{H}$ trace represent $90^{\circ}$ and $180^{\circ}$ hard RF pulses respectively, while the shaped pulse represents a soft $180^{\circ}$ pulse. The incremented delays for the $J$-evolution and for the reconstruction of the pure shift interferogram are denoted as $\tau_{1} / \tau_{3}$ and $t_{1}$ respectively. $G_{1}, G_{3}$ and $G_{4}$ are CTP field gradient pulses and $G_{2}$ is a weaker gradient pulse for spatial encoding. Full experimental details are given in the experimental section of the ESI. $\dagger$ 


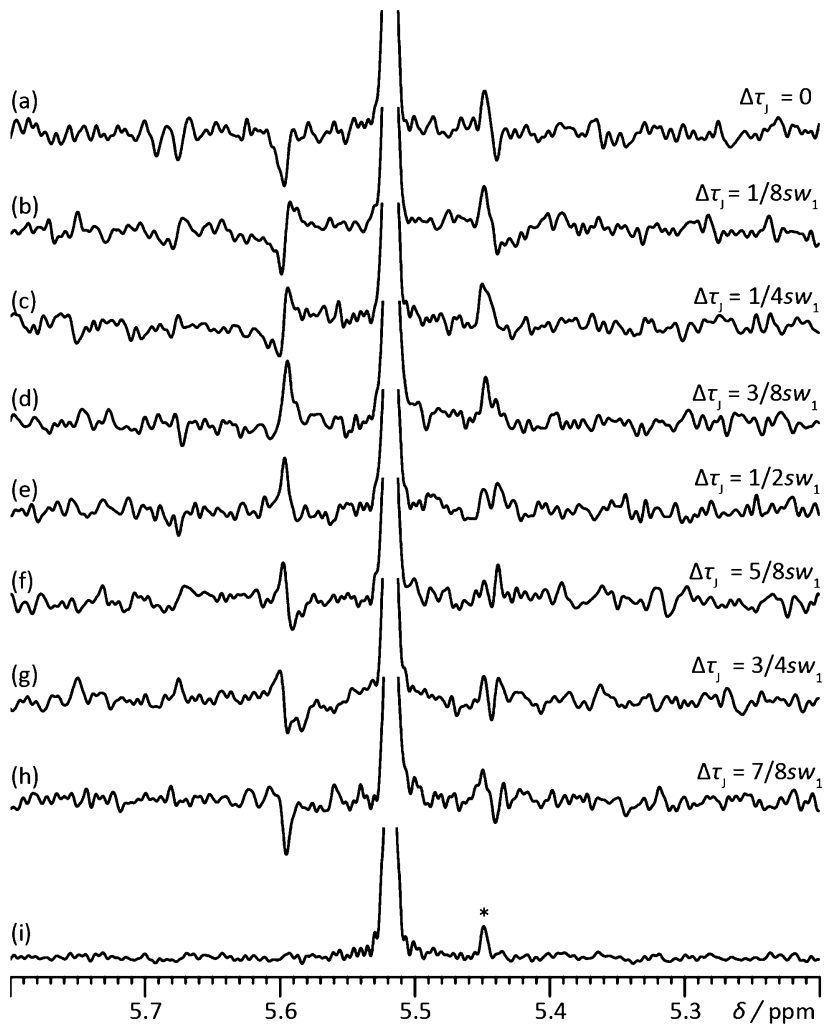

Fig. 3 Illustration of the cyclic phase rotation of the sidebands for $\mathrm{H}_{6}$ of rosuvastatin in the sample used for Fig. 1 . Here, the right hand first-order side sideband overlaps with a BEM signal $\left.{ }^{*}\right) . \Delta \tau_{j}$ indicates the incremented $\mathrm{J}$ evolution time. The modulation phase changes in spectra (a) to (h) in steps of $+45^{\circ}$, starting from $-180^{\circ}$. The sum of spectra $(a-h)$ is shown in (i).

In this way all $N$ interferograms experience the same $T_{2}$ weighting. The process is summarised in Fig. S6 of the ESI, $\dagger$ which shows the 8 different modulation patterns for $N=8$. The corresponding experimental data are shown in Fig. $3 \mathrm{a}$ to $\mathrm{h}$; averaging the 8 spectra leads to a clean spectrum with sidebands suppressed to order 7. Since the sidebands decrease rapidly in amplitude with increasing order, ${ }^{16}$ it is rarely necessary to go beyond $N=3$.

At first sight the new method would appear to be very timeconsuming, but in practice this is not the case. The sensitivity of the new method is almost identical to that of the parent experiment, the only difference is a small extra penalty incurred in $T_{2}$ relaxation during the period $2 \tau_{2}$. To acquire a pure shift spectrum in which chunking sidebands are visible above the noise will almost always require significant time averaging, so the minimum experiment duration is unchanged, and even the simplest possible implementation (a 2-step SAPPHIRE suppression) will yield $90 \%$ of the benefit (Fig. S8, ESI $\dagger$ ). Apart from the very small extra $T_{2}$ weighting, under high resolution conditions the quantitative character of SAPPHIRE is essentially the same as that of the parent pure shift method (Zangger-Sterk in the example shown): the intensity of the centreband is unaffected by the suppression of the sidebands.

The extra echo in Fig. 2 can be dispensed with if desired, reverting to the basic framework of the standard Zangger-Sterk sequence of Fig. S1 (ESI $\dagger$ ). Instead of respectively decrementing and incrementing the delays $\tau_{1}$ and $\tau_{3}$ of the sequence of Fig. 2, the delay $\tau_{1}$ of Fig. S1 (ESI $\dagger$ ) is decremented until it reaches zero, at which point the hard $180^{\circ}$ pulse echo and the soft pulse echo are interchanged and $\tau_{1}$ is incremented until the modulation cycle is complete. If the sequence of Fig. S1 (ESI $\dagger$ ) is used it is necessary to adjust the phase of the soft $180^{\circ}$ pulse to match exactly that of the hard $180^{\circ}$ pulse, in order to avoid a phase discontinuity between the two sequence variants. ${ }^{8}$

The results presented here were acquired using the original Zangger-Sterk J-refocusing method. Exactly the same approach can be used with other $J$-refocusing sequence elements such as PSYCHE $^{11}$ or band-selection ${ }^{9,17}$ as shown in Fig. S10 and S11 of the ESI. $\dagger$ The logic of the SAPPHIRE method should also be independent of whether data are acquired in interferogram or real-time mode.

In direct-domain pure shift experiments there is a tension between sensitivity and spectral purity when choosing the parameter $\mathrm{sw}_{1}$, which determines the data chunk duration $1 / \mathrm{sw}_{1}$. Residual $J$ modulation sidebands increase rapidly as $\mathrm{sw}_{1}$ decreases, so it is usual to choose a value of $\mathrm{sw}_{1}$ large compared with the maximum multiplet width of interest. Practical experiments typically use $\mathrm{sw}_{1}$ values of the same order as the $39 \mathrm{~Hz}$ used for Fig. 1 and 3. With the SAPPHIRE method it is possible to reduce $\mathrm{Sw}_{1}$ substantially, to values that would normally give rise to unacceptably strong chunking sidebands, as illustrated by the results in Fig. S9 of the $\mathrm{ESI}^{\dagger}$ with $\mathrm{Sw}_{1}$ values of 10 and $20 \mathrm{~Hz}$. At first sight it is very tempting to make use of the two- or four-fold time saving this would allow. Unfortunately while the sidebands are very efficiently suppressed, a price is paid for the time saving in distorted signal intensities, wide multiplets giving slightly weaker pure shift singlets.

One welcome by-product of chunking sideband suppression is a reduction in strong coupling artefacts. All pure shift methods fail to a greater or lesser extent when spins are strongly coupled. ${ }^{18}$ Where this failure leads to signal discontinuities between data chunks, the artefacts that result are periodic, as for example around 2 ppm in Fig. $1 \mathrm{~b}$ and $\mathrm{c}$ and in Fig. S12 of the ESI. $\dagger$ SAPPHIRE averaging suppresses these periodic artefacts, leading to the much cleaner result in Fig. 1d. A further improvement could result if this is combined with the recently-published triple spin echo PSYCHE sequence. ${ }^{19}$

Over 70 papers have been published to date on directdomain pure shift methods. In every case, the use of chunked data acquisition, whether in real time or in pseudo-2D (interferogram) mode, leads to periodic artefacts. These chunking sidebands can cause significant problems in the analysis of low-level components of mixtures (and indeed of other low-level signals such as those of end groups and branch points in polymers). The novel approach introduced here offers the possibility of acquiring ultraclean, high dynamic range ${ }^{1} \mathrm{H}$ pure shift spectra by suppressing these artefacts, whether in interferogram or in real-time pure shift experiments, without affecting the quantitative character of the measurement.

This work was supported by AstraZeneca and by the Engineering and Physical Sciences Research Council (grant numbers 
EP/M013820 and EP/N033949). All raw experimental data, pulse sequence code and processing software can be downloaded from DOI: $10.15127 / 1.309229$.

\section{Notes and references}

1 R. W. Adams, eMagRes, John Wiley \& Sons, Ltd, 2007, DOI: 10.1002/ 9780470034590.emrstm1362.

2 L. Castañar and T. Parella, Magn. Reson. Chem., 2015, 53, 399-426.

3 K. Zangger, Prog. Nucl. Magn. Reson. Spectrosc., 2015, 86-87, 1-20.

4 G. Dal Poggetto, L. Castañar, G. A. Morris and M. Nilsson, RSC Adv., 2016, 6, 100063.

5 M. Foroozandeh, L. Castañar, L. G. Martins, D. Sinnaeve, G. Dal Poggetto, C. F. Tormena, R. W. Adams, G. A. Morris and M. Nilsson, Angew. Chem., Int. Ed., 2016, 55, 15579-15582.

6 M. Nilsson and G. A. Morris, Chem. Commun., 2007, 933-935, DOI: 10.1039/B617761A.

7 A. Lupulescu, G. L. Olsen and L. Frydman, J. Magn. Reson., 2012, 218, 141-146.

8 K. Zangger and H. Sterk, J. Magn. Reson., 1997, 124, 486-489.
9 R. W. Adams, L. Byrne, P. Kiraly, M. Foroozandeh, L. Paudel, M. Nilsson, J. Clayden and G. A. Morris, Chem. Commun., 2014, 50, 2512-2514.

10 L. Castañar, P. Nolis, A. Virgili and T. Parella, Chem. - Eur. J., 2013, 19, 15472-15475.

11 M. Foroozandeh, R. W. Adams, N. J. Meharry, D. Jeannerat, M. Nilsson and G. A. Morris, Angew. Chem., Int. Ed., 2014, 53, 6990-6992.

12 A. J. Pell, R. A. E. Edden and J. Keeler, Magn. Reson. Chem., 2007, 45, 296-316.

13 J. A. Aguilar, S. Faulkner, M. Nilsson and G. A. Morris, Angew. Chem., Int. Ed., 2010, 49, 3901-3903.

14 J. Mauhart, S. Glanzer, P. Sakhaii, W. Bermel and K. Zangger, J. Magn. Reson., 2015, 259, 207-215.

15 E. Kupče, R. Freeman, G. Wider and K. Wüthrich, J. Magn. Reson., Ser. A, 1996, 122, 81-84.

16 L. Kaltschnee, K. Knoll, V. Schmidts, R. W. Adams, M. Nilsson, G. A. Morris and C. M. Thiele, J. Magn. Reson., 2016, 271, 99-109.

17 L. Castañar, M. Pérez-Trujillo, P. Nolis, E. Monteagudo, A. Virgili and T. Parella, ChemPhysChem, 2014, 15, 854-857.

18 M. J. Thrippleton, R. A. E. Edden and J. Keeler, J. Magn. Reson., 2005, 174, 97-109.

19 M. Foroozandeh, R. W. Adams, P. Kiraly, M. Nilsson and G. A. Morris, Chem. Commun., 2015, 51, 15410-15413. 